\title{
Herzschutz aus der Natur
}

_ Stress und Sorgen gehen zu Herzen. Das menschliche Immunsystem reagiert auf akuten und chronischen psychische Stress u. a. mit der Aktivierung proinflammatorischer Zytokine, die wiederum Risikofaktoren für das spätere Auftreten einer koronaren Herzerkrankung (KHK) darstellen. Soziale und psychische Faktoren müssen daher bei der Einschätzung des KHK-Risikos berücksichtigt werden.

Ganzheitliche Behandlungsoptionen können dazu beitragen, die physiologischen Rhythmen zu synchronisieren und damit der Entwicklung manifester Erkrankungen vorzubeugen. Neben der individuellen Änderung des Lebensstils gehören hierzu nicht-medikamentöse wie auch arzneiliche Behandlungsformen.

Beispielsweise hat sich die Musiktherapie als Behandlungsverfahren in vielen deutschen Kliniken etabliert und wird zunehmend auch in die wissenschaftlichen Therapieleitlinien aufgenommen. Mit Cardiodoron (ein Extrakt aus Schlüsselblumen, Bildenkrauttinktur und Eselsdistel) und Neurodoron ${ }^{\circ}$ (Kalium phosphoricum D6, Ferrumquarz D2 und Aurum metallicum praeparatum D10) stehen zudem Arzneimittel aus der anthroposophischen Medizin zur Verfügung, die das Herz unterstützen und die Stressresistenz erhöhen können.

\section{Hier steht eine Anzeige.}

\author{
Springer
}

\section{Akute Pharyngitis}

\section{Nebenwirkungsarme Schmerzlinderung}

_ Bei starken bis sehr starken Halsschmerzen bieten Lutschtabletten mit Benzydamin (neo-angin ${ }^{\circ}$ ) die Möglichkeit einer gezielten lokalen Therapie. Der Wirkstoff penetriert Haut und Schleimhaut gut und reichert sich im entzündeten Gewebe an. Dadurch werden auch bei lokaler Anwendung wirksame Konzentrationen am Ort der Entzündung erreicht. Benzydamin gehört zur Klasse der NSAR, bekämpft die Entzündung jedoch über die Hemmung der Freisetzung pro-inflammatorischer Zytokine. In therapeutischer Dosis werden weder die Cyclo- noch die Lipoxygenase gehemmt, sodass die Prostaglandinsynthese nicht beeinflusst wird. Die bekannten NSAR-typischen gastrointestinalen Nebenwirkungen wie Übelkeit, Erbrechen, Durchfälle, Ulzera und/oder Blutungen treten daher bei der Anwendung von Benzydamin in therapeutischen Dosen nicht auf.

Red.

- Nach Informationen von MCM 